\title{
Thorax measurement and analysis using electrical impedance tomography
}

\author{
David Krčmařík ${ }^{1}$, Michal Petrů로 Jan Koči ${ }^{3}$ \\ Institute for Nanomaterials, Advanced Technologies and Innovation, Technical University of Liberec, \\ 46117 Liberec, Czech Republic \\ ${ }^{1}$ Corresponding author \\ E-mail: ${ }^{1}$ david.krcmarik@tul.cz, ${ }^{2}$ michal.petru@tul.cz, ${ }^{3} j a n . k o c i @ t u l . c z$
}

Received 30 August 2019; accepted 6 September 2019 DOI https://doi.org/10.21595/vp.2019.20986

Check for updates

Copyright $(C) 2019$ David Krčmařik, et al. This is an open access article distributed under the Creative Commons Attribution License, which permits unrestricted use, distribution, and reproduction in any medium, provided the original work is properly cited.

\begin{abstract}
The article deals with a novel method of visualizing interior of an object based on the measurements made on the boundary. Although an electrical impedance tomography is well established in areas where reference measurement can be easily made (difference method), it is still rather a theoretical approach for areas where reference cannot be taken (mainly in medicine). We have made a thorax measurement using difference method. The results show that electrical impedance tomography can provide valuable information for thorax visualization.
\end{abstract}

Keywords: difference, algorithm, tomography, thorax.

\section{Introduction}

Recently a lot of attention was focused on utilization of the Electrical Impedance Tomography (EIT) in medical care. EIT is a technique of visualizing interior of an object based on electrical measurements made on the surface. Although there are already other well-established techniques like a magnetic resonance imaging (MRI) or a computed tomography (CT) they are both not suitable for a real-time monitoring at the bedside. Moreover, EIT offers less expensive, non-ionizing approach. The main drawback of EIT is mainly that it provides images which are blurred and have spatial resolution lower than MRI or CT. The spatial resolution is at best limited approximately by the distance between electrodes.

In medicine EIT can be generally used for phenomena where an impedance change over time is encountered. For example, a successful bladder filling [1] surveillance, a stomach gastric emptying [2] and of course lung ventilation [3] and a cardiac perfusion [4] in the area of thorax is reported. In this report we will focus on imaging of ventilation and perfusion [5].

There are basically two types of EIT: absolute and difference. The absolute one is very vulnerable to mismatch in electrode positioning, not exactly specified geometry of the measured object or inaccurately known contact impedance. This concept is for the time being developed mainly on phantoms although few measurements on real targets were already made [6]. On the other hand, to circumvent such problems a difference measurement can be made when a reference is taken at the begging of the measurement. This is clearly unsuitable for thoracic measurements. To overcome such a limitation of difference imaging a concept of an average impedance over a certain time window as a reference [3] was proposed or computation of local standard deviations at each pixel and plotting them at the corresponding image position [3] was used. In this paper we tried another approach of taking reference during a deep expiration of a subject.

The main objective of EIT imaging of thorax is nowadays an individual and real-time adjustment of mechanical ventilation for patients with problems with breathing such as acute respiratory distress syndrome (ARDS) $[7,8]$.

\section{Materials and methods}

As it is visible from previous section EIT is a promising way of reconstructing cross-sectional slices of an object based on boundary measurements. EIT is in fact a device which consists from 
a precise current source and a very accurate voltmeter. The electrodes on the boundary of a vessel (or a human body in our case) are driven by the current source (one electrode is a source and a different one is a sink) and the voltage measurements are made on the remaining electrodes (Fig. 1). Clearly when an object is inserted to the test vessel which was not there while taking reference (Fig. 1(a)) the voltage at the electrode E (Fig. 1(b)) differs from the voltage taken at the time of taking the reference.

There is also an approach when several electrodes are driven at the same time [9] but this one is rarely used (this approach delivers very precise measurements which can be then used even for absolute imaging) because it is very complex. There are many modifications of tomographic systems. They usually differ in the hardware used and sensing strategy.

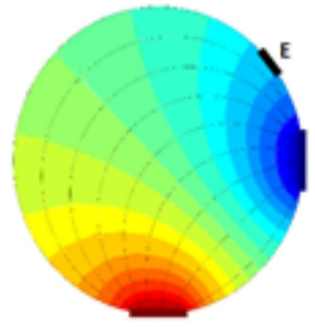

a)

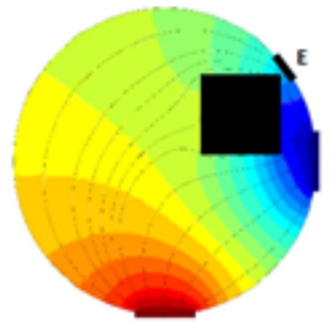

b)

Fig. 1. Principle of voltage changes on an arbitrary electrode E, a) vessel with fluid, b) a square cross-sectional object is inserted into the vessel with fluid.

\subsection{Sensing strategy}

The most common system in use is using Sheffield strategy (adjacent) when adjacent electrodes are driven by current and at the same time different adjacent electrodes are measured for the difference induced voltage. For a system with one plane and 16 electrodes (this system will be considered in this paper) this results for total of 104 independent measurements. This was the first successful system and because of historical reasons the system is still widespread even though it was shown that other strategies exercise inherently better results [10]. From [10] it follows that the best measurement strategy is stimulation and sensing in a way when electrodes are nearly opposite but not exactly opposite. This strategy is also called [1 7] strategy or "skip 5" (electrodes 1 and 7 are driven by current and electrodes 2 and 8 are sensed etc.). Nevertheless, we have examined all three often discussed strategies in our experiment to verify the best method. A circular test vessel was filled with tapped water and on the bed of the vessel was thick sediment of sand. At this point we took a reference of the measuring plane above the sediment and started to turn the vessel upside down. Two positions were selected for taking a measurement: the first one when sand formed at the sensing plane small deposit (Fig. 2 - first row) and the second one when the sand filled nearly the whole area of the sensing plane (Fig. 2 - second row). Results after FEM processing are visible in Fig. 2(a, d) for adjacent strategy, in Fig. 2(b, e) for opposite strategy and in Fig. 2(c, f) for [1 7] strategy. It is visible that adjacent strategy reliably represents a real scenario, opposite stimulation (pattern [1 9]) and sensing have problems with mirroring the object (the images are axial symmetric) and finally the so-called optimal strategy (pattern [1 7]) does not correspond to the real scenario. Based on this experiment we have decided to use traditional adjacent strategy further on since it provided by far the most reliable results.

In the case of adjacent and optimal pattern there are 104 measurement whereas for opposite there are only 28 measurements.

The main EIT advantages (compared to most common computer tomography - CT - scans) are mainly: low cost, high acquisition frequency, small size and non-ionizing approach. The drawback of EIT which is low spatial resolution is caused mainly by the fact of badly-posed problem [11] because of inevitable errors during measurement and due to quantization. 


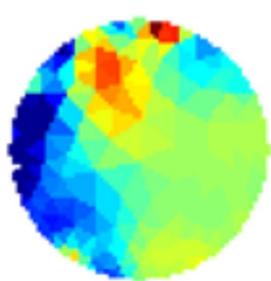

a)

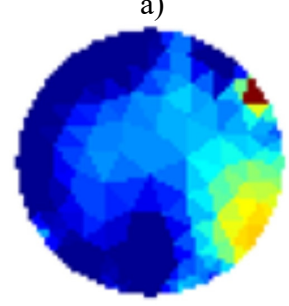

d)

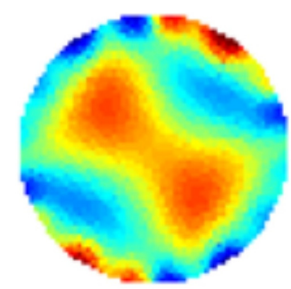

b)

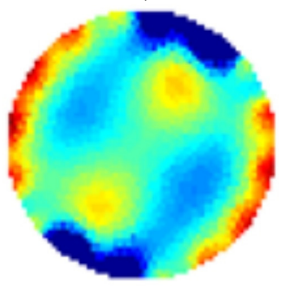

e)

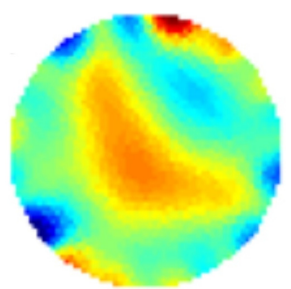

c)

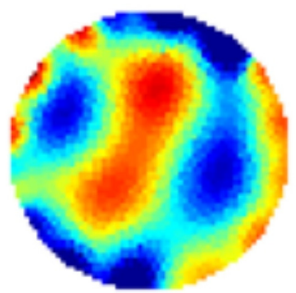

f)

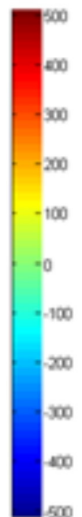

Fig. 2. Comparison of results from different stimulation and sensing strategies; a) and d) adjacent, b) and e) opposite and c) and f) optimal [1 7], the first row corresponds to small deposit of sand and the second row corresponds to large deposit of sand at the level of sensing plane

\subsection{Computation}

The theory behind is very complex. Basically, a finite element method (FEM) is used for image reconstruction. Fortunately, there is an open source library for Matlab (an engineering computational software) called EIDORS [12] which contains functions for various measured data processing in a cross-sectional area of the object. Since the measurement contains noise from various sources [11] it is necessary to use some kind of regularization [11]. Basically, the initial problem after linear approximation can be stated as:

$J \times x=b$,

where $J$ is a Jacobian (or a sensitivity matrix) coming from FEM analysis reconstruction, $x$ is a sought column vector of unknowns and $b$ is a column vector of measured data on the boundary. Since we are using difference imaging the $\mathrm{x}$ has the meaning of a change in conductivity and $\mathrm{b}$ has to be replaced by $b_{2}-b_{1}$ where $b_{1}$ is the measurement taken as the reference and $b_{2}$ is the measurement taken at the time $t>0$. The Eq. (1) appended with regularization can be solved either by the way of quadratic (most common - 2-norm) or linear (more robust since the error is powered by $1-1$-norm) norm. The norms can be applied both for data and model - see [13]. The overall problem can then be stated as a minimalization problem with properly chosen hyperparameter $\lambda$ :

$\hat{x}=\underset{x}{\operatorname{argmin}}\|W(b-J x)\|^{n_{d}}+\|\lambda L x\|^{n_{m}}$,

where $\hat{x}$ is the solution, $W$ is a weighting matrix, $L$ is the penalty matrix (for the simplest Tikhonov prior it is the identity matrix) and the whole second term is the so-called regularization term. The data norm is denoted as nd and the image norm is denoted as $\mathrm{nm}-$ see $[11,13]$. The hyperparameter has the effect of damping any large oscillation but it has the side effect of smoothing.

EIDORS provide several algorithms (the most common is "Gauss-Newton one step") and several prior information. EIDORS also provides visualization of sensitivity maps where one can see which parts of the object are more sensitive to impedance changes based on the sensing strategy used. It is important to emphasize the role of the hyperparameter $\lambda$ in Eq. (2) since it 
controls the proportion between regularization (in other words kind of filtering) and proper solution based on measured noisy data. The use of L-curve technique [14] is mostly constricted because of very imperceptible curvature part of the graph.

\section{Results and discussion}

There were many attempts to introduce the EIT methodology into clinical applications. One of them is for example designing an active electrode belt for the sake of ease of placing electrodes and minimizing errors [15]. Also, several rather experimental measurements of thorax were reported for pigs and even for humans Anyway monitoring lungs is very challenging because it is not possible to use the differential approach. The main advantage of the differential approach is that it minimizes the effect of contact impedances of electrodes and to certain level also minimizes not precisely located electrodes.

For the case of lungs, it is impossible to take the reference and hence using true differential approach. Regardless of this we have used "pseudo-differential" approach - see [15]. In our case the volunteer has strived to exhale as much air as possible and at that moment we have taken a reference. Then during the measurement, the volunteer has taken deep breath for two times and in between he again exhaled as much as possible. A commercial p2+ EIT system from ITS, England was used. The average resistivity measured across all the calculated data points in the cross section is visible from Fig. 3(a). The graph can be used for estimation of end-expiratory lung volume (EELV) from end-expiratory lung impedance (EELI). It was proven that a linear dependence exists between EELI and EELV [16].

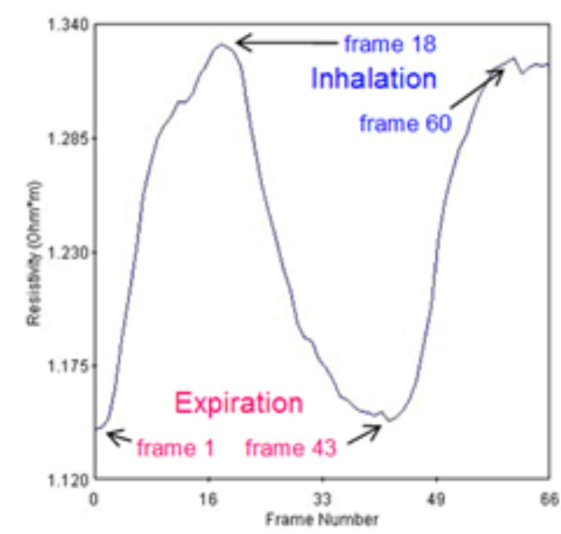

a)

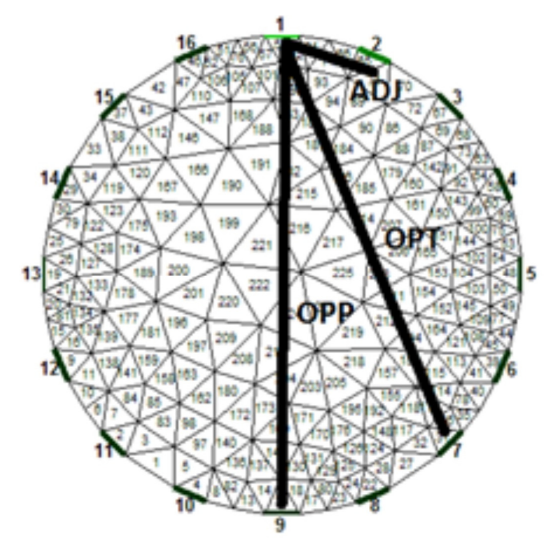

b)

Fig. 3. a) Average resistivity measurements for a volunteer exhaling as much as possible and taking deep breath, b) electrode assignment for 16-electrode system

The results using 1-norm (1-norm applied only for image part - see Eq. (2)) are depicted in Fig. 4. When the volunteer had taken deep breath (Fig. 4(b) and 4(d)) it is clearly visible the outer of the lungs in blue since the lungs had absorbed the air which is less conductive than human tissue. The electrical current cannot flow through the alveoli but only around them through the alveolar septa. When the alveolar volume increases the average current pathways stretch and prolong resulting in increase electrical resistance. The solution of Eq. (2) is then:

$x=\left(J^{T} J+\lambda I\right)^{-1} J^{T} \times b$,

where $J^{T}$ stands for transpose of $J$.

What is also interesting is that on the left side of the thorax is visible slightly more conductive region (Fig. 5(b) and 5(d)). We think this can be partially attributed to heart and aorta. Of course, several errors are visible on the circumference of the thorax mainly due to electrodes nearby. For 
proper analysis it would be necessary taking into consideration also varying circumference of the thorax between inhalation and exhalation [13]. The circumference of the volunteer was about $96 \mathrm{~cm}$ in the rest - since we have used 16 electrode system the electrodes were placed $6 \mathrm{~cm}$ from each other. The circumference grew by about $10 \mathrm{~cm}$ when taking deep breath. The circular electrodes (diameter $1 \mathrm{~cm}$ ) were placed approximately on the level of the 5 th rib from the bottom of the thorax and attached to the body via a tape. A conducting gel was used in order to lower contact impedance. The current used was $15 \mathrm{~mA}$. The measurement strategy used was adjacent. Hyperparameter was chosen to be 0.0002 and the iterations were kept low for the reason of speed - we have used 2 iterations.

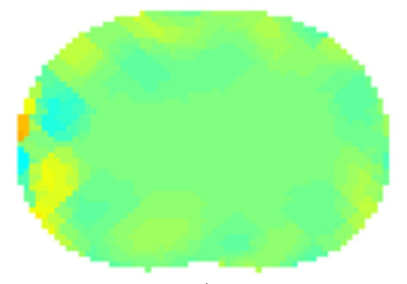

a)

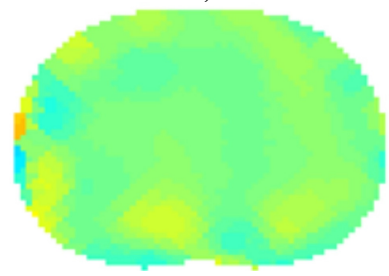

c)

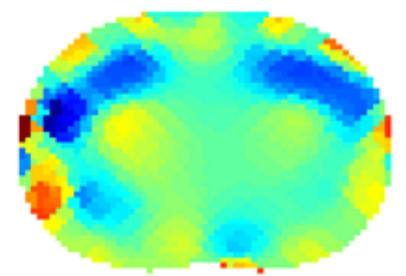

b)

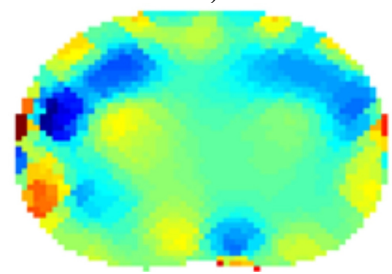

d)

Fig. 4. Results of volunteer breathing a) exhaling-frame 1, b) deep breath-frame 18, c) exhaling-frame $43, \mathrm{~d}$ ) deep breath-frame 60 (bottom is spine)

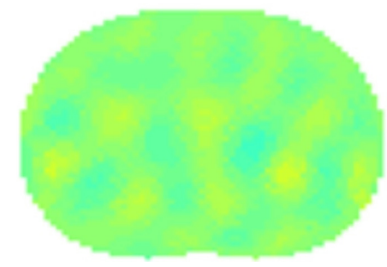

a)

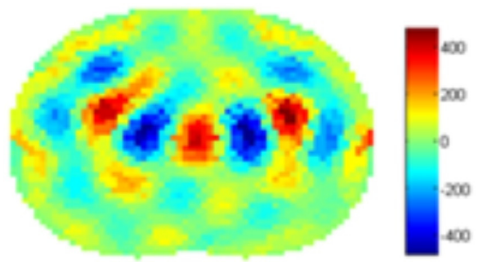

b)

Fig. 5. Results of volunteer breathing a) exhaling (frame 1), b) deep breath (frame 18

We have processed the measured data also using standard 2-norm approach. The results are on Fig. 5. The Gauss-Newton one step algorithm was used for reconstruction

For the case of Noser prior the right and left regions of lungs are more clearly visible but with very severe errors in the middle of each lung and in the middle of the trunk. We suspect that this can be attributed to algorithm artifacts. For the case of Laplace prior the results are very similar to the results of 1-norm presented in Fig. 4.

\section{Conclusions}

We have experimentally shown measurement of lungs on a volunteer. Since absolute imaging is very challenging, we have decided to use pseudo-differential imaging providing that reference image was taken when the volunteer exhaled as much air as possible. The results correspond well with expected phenomena. We were surprised to see the difference between the left and right lungs and we attribute this to heart and aorta. The standard deviation map can nicely visualize main 
activity inside the thorax cross-section. The results provided by EIT can be applied into clinical practice where a real-time monitoring of the patient at the bedside can help with individual adjustment of mechanical ventilation.

\section{Acknowledgements}

The result was obtained through the financial support of the Ministry of Education, Youth and Sports of the Czech Republic and the European Union (European Structural and Investment Funds - Operational Programme Research, Development and Education) in the frames of the project "Modular Platform for Autonomous Chassis of Specialized Electric Vehicles for Freight and Equipment Transportation", Reg. No. CZ.02.1.01/0.0/0.0/16_025/0007293.

\section{References}

[1] He W., Ran P., Xu Z., Li B., Li S. N. A 3D visualization method for bladder filling examination based on EIT. Computational and Mathematical Methods in Medicine, Vol. 1012, 2012, p. 528096.

[2] Adler A., Guardo R., Berthiaume Y. Imaging of gastric emptying with electrical impedance tomography, http://www.sce.carleton.ca/faculty/adler/publications/1994/adler-cmbs94-gastricemptying.pdf.

[3] Hahn G., Sipinkov I., Aaisch F., Hellige G. Changes in the thoracic impedance distribution under different ventilatory conditions. Physiological Measurement, Vol. 16, 1995, p. 161-173.

[4] Deibele J. M., Luepschen H., Leonhardt S. Dynamic separation of pulmonary and cardiac changes in electrical impedance tomography. Physiological Measurement, Vol. 29, Issue 6, 2008, p. 1-14.

[5] Leonhardt S., Lachmann B. Electrical impedance tomography: the holy grail of ventilation and perfusion monitoring? Intensive Care Medicine, Vol. 38, Issue 12, 2012, p. 1917-1929.

[6] Hahn G., Just A., Dudykevych T., Frerichs I., Hinz J., Quintel Hellige M. G. Imaging pathologic pulmonary air and fluid accumulation by functional and absolute EIT. Physiological Measurement, Vol. 27, Issue 5, 2006, p. 187-198.

[7] Wolf G. K., Gomez Laberge C., Rettig J. S., Vargas S. O., Smallwood C. D., Prabhu S. P., Vitali S. H., Zurakowski D., Arnold J. H. Mechanical ventilation guided by electrical impedance tomography in experimental acute lung injury. Critical Care Medicine, Vol. 41, Issue 5, 2013, p. 1296-1304.

[8] Luepschen H., van Riesen D., Beckmann L., Hameyer K., Leonhardt S. Modeling of fluid shifts in the human thorax for electrical impedance tomography. IEEE Transactions on Magnetics, Vol. 44, Issue 6, 2008, p. 1450-1453.

[9] Isaacson D., Mueller J. L., Newell J. C., Siltanen S. Reconstructions of chest phantoms by the D-bar method for electrical impedance tomography. IEEE Transactions on Medical Imaging, Vol. 23, Issue 7, 2004, p. 821-828.

[10] Adler A., Gaggero P. O., Maimaitijiang Y. Adjacent stimulation and measurement patterns considered harmful. Physiological Measurement, Vol. 32, Issue 7, 2011, p. 731-744.

[11] Holder D. S. Electrical Impedance Tomography: Methods, History and Applications. Taylor and Francis, 2010.

[12] Adler A., Lionheart W. R. B. Uses and abuses of EIDORS: an extensible software base for EIT. Physiological Measurement, Vol. 27, Issue 5, 2006, p. 25-42.

[13] Grychtol B., Adler A. FEM electrode refinement for electrical impedance tomography. 35th Annual International Conference of the IEEE Engineering in Medicine and Biology Society (EMBC), Osaka, Japan, 2013.

[14] Hansen P. C. The L-Curve and Its Use in the Numerical Treatment of Inverse Problems. Computational Inverse Problems in Electrocardiology, 2000.

[15] Gaggero P. O. Miniaturization and Distinguishability Limits of Electrical Impedance Tomography for Biomedical Application. Ph.D. Thesis, Faculty of Science of the University of Neuchatel, Switzerland, 2011.

[16] Grivans C., Lundin S., Stenqvist O., Lindgren S. Positive end-expiratory pressure-induced changes in end-expiratory lung volume measured by spirometry and electric impedance tomography. Acta Anaesthesiol Scand, Vol. 55, Issue 9, 2011, p. 1068-1077. 